\title{
$T$ Parity and the Littlest Higgs
}

\author{
Ian Low \\ Jefferson Physical Laboratory, Harvard University, Cambridge, MA 02138
}

\begin{abstract}
We construct $T$-parity invariant extensions of the littlest Higgs model, in which only linear representations of the full symmetry group are employed, without recourse to the non-linear representations introduced by Coleman, Callan, Wess, and Zumino (CCWZ). These models are based on the symmetry breaking pattern $S U(5)_{l} \times H_{r} / S O(5)$, where $H_{r}$ can be $S O(5)$ or other larger symmetry groups. The structure of the models in the $S U(5)_{l}$ sector is identical to the littlest Higgs model based on $S U(5) / S O(5)$. Since the full symmetry group is realized linearly, these models can be thought of as possible UV extensions of the $T$-invariant model using non-linear representations via CCWZ, with whom they share similar low energy phenomenology. We also comment on how to avoid constraints from four-fermion operators on $T$-invariant models with or without CCWZ construction. The electroweak data therefore place a very weak bound on the symmetry breaking scale, $f \geq 450 \mathrm{GeV}$.
\end{abstract}




\section{INTRODUCTION}

With the Large Hadron Collider (LHC) at CERN setting to start running in 2007, we are getting closer to unravel the mystery of the electroweak symmetry breaking. In the standard model the electroweak symmetry is broken by the vaccum expectation value of a scalar particle, the Higgs particle, whose mass-squared receives quadratically divergent contribution from the UV physics at the quantum level. In order to stabilize the electroweak scale naturally, new physics is expected at around $1 \mathrm{TeV}$, which is the energy scale that will be directly probed by the LHC. Therefore much effort has been dispensed to construct models for new physics at the $\mathrm{TeV}$ scale, which will be discernible by experiments in the coming decade.

On the other hand, the standard model as we know today has been very successful in confronting current experimental data. Precision measurements in the last ten years reveal very little deviation from the prediction of the standard model. In the low energies we can parametrize the effect of new physics by a set of higher dimensional operators involving the standard model fields only [1], whose sizes are then constrained by the precision electroweak measurements 2]. The statement that current data agree with the standard model predictions to a high degree means the coefficients of these higher dimensional operators are all very small. This in turn implies the scales of new physics, which suppress the higher dimensional operators, are very large if one assumes all the dimensionless numbers are order unity. The most loosely constrained operators are those consistent with the (approximate) symmetries of the standard model. Even for these operators current experiments already suggest suppressions by energy scales as high as $5-10 \mathrm{TeV}$ [3]. A naive interpretation would then be that new physics doesn't come in until $5-10 \mathrm{TeV}$, creating a tension with the naturalness principle which expects new physics at $1 \mathrm{TeV}$.

There are two opposite attitudes we can take toward this "little hierarchy problem." One is to abandon the naturalness principle and try to make peace with the fine-tuning. This approach has lead to the prediction of the cosmological constant by the anthropic principle [4], as well as interesting models on physics beyond the standard model such as the split supersymmetry [5]. Nevertheless, naturalness consideration as a principle has been successful in predicting the existence of new physics in the past. One example is the self-energy of the electron (see, for example, Refs. 66, 7] ), which classically is linearly divergent due to the $1 / r$ Coulomb potential. Naturalness principle then expects new physical degree of freedom at the scale $m_{e}$, the electron mass. Indeed at the energy scale $m_{e}$ we need to take into account the creation of electron-positron pair out of the vaccum. After including the contribution from the positron, the electron self-energy is only logarithmically sensitive to the UV physics. Another example in which the naturalness consideration works as expected is the low energy physics of QCD [7]. The charged $\pi$ mesons, being pseudo-Nambu-Goldstone bosons, receive quadratically divergent contribution to their mass from photons at one loop. If the $\pi$ masses are to be in the $100 \mathrm{MeV}$ range naturally, new degrees of freedom should start showing up before $1 \mathrm{GeV}$. In this case, new physics such as the $\rho$ and $a_{1}$ mesons do come in below the scale suggested by naturalness. Therefore it is perhaps not surprising that the second attitude, that is to take naturalness seriously, has been the driving force in theorizing physics beyond standard model in the last couple decades.

Given that there are very few hints from experiments on the new physics at the $\mathrm{TeV}$ scale, it is important to seek a resolution of the little hierarchy problem without abandoning the naturalness principle. One simple model-independent solution is the existence of a new 
$Z_{2}$ symmetry acting only on the new particles at the TeV scale [8, 9]. Examples of this $T$-parity include the $R$-parity in the supersymmetric standard model and the KK-parity in models with universal extra-dimensions. On the model building side, a new class of theories dubbed the little Higgs theories was proposed [10, 11, 12] with solving the little hierarchy problem in mind, which stabilizes the electroweak scale naturally while raising the cutoff of the theory to $10 \mathrm{TeV}$, beyond the probe of current electroweak data. There are many variants of the little Higgs models 13, 14, 15, 16, 17, 18], as well as some examples of UV extensions above $10 \mathrm{TeV}$ 19, 20], and extensive phenomenological studies have been performed [21, 22, 23, 24, 25, 26, 27, 28, 29]. It turned out the impact of little Higgs models on the precision observables is quite model dependent; some requires raising the cutoff higher than $10 \mathrm{TeV}$, thus re-introducing the fine-tuning, and some do not. In Refs. [9, 30] it is shown that it is possible to combine the idea of $T$-parity with the little Higgs theories, and thus eliminate the precision electroweak constraints on a large class of little Higgs models. Moreover, the lightest $T$-odd particle, the LTP, is stable and massive in the desirable range to be a good dark matter candidate.

There are in fact many ways to implement $T$-parity on little Higgs models. In Ref. 9] a three-site moose model was constructed in which the $T$-parity is a variant of the $Z_{2}$ reflection symmetry between two sites. The third site then remains neutral under the $T$-parity. Later it was realized that $T$-parity can be consistently implemented on any non-linear sigma model based on a symmetric coset space $G / H$. T-parity invariant models of the minimal moose type [11] and the littlest Higgs type [12] are given in which non-linear representations introduced by Callan, Coleman, Wess, and Zumino (CCWZ) 31, 32] are utilized to assign the representations of the fermions [30]. While from the low energy perspective the formalism of CCWZ is the natural one to consider, it makes no reference to what the possible UV extensions may be. In this regard the three-site moose model, where all the matter is assigned to linear representations of the full symmetry group $G$, is more straightforward to UV-complete. ${ }^{1}$ Then the two-site minimal moose model can be considered as a descendent of the three-site model by integrating out the neutral third site.

In this paper we consider extensions of the $T$-invariant models of the littlest Higgs type, without recourse to the machinery of CCWZ, for which it may be more straightforward to imagine possible UV completions [33]. Similar to the minimal moose model, these extensions are achieved by extending the global symmetry group, in this case to $S U(5)_{l} \times H_{r} / S O(5)_{v}$, where $H_{r}$ is a group containing $S O(5)$ and the unbroken subgroup is the vectorial $S O(5)$. In section II we consider the case with the minimal group structure with $H_{r}=S O(5)$. Additional fermions need to be introduced as well. A somewhat larger group structure with $H_{r}=S U(5)$ is considered in section III, where less number of fermionic degree of freedom is required. In both cases the $S U(5)_{l}$ sector is identical to the $S U(5) / S O(5)$ littlest Higgs, thus distinguishing them from the minimal moose type model where at least four link fields are necessary. Furthermore, the extra scalar and vector particles that come with the extended global symmetry can all be made heavy at around $10 \mathrm{TeV}$ and integrated out of the spectrum. In the last section we comment on the implications of our constructions on the $T$-invariant models using non-linear representations of CCWZ and explain how to avoid constraints from four-fermion operators on the model proposed in Ref. [30].

\footnotetext{
${ }^{1}$ One trivial way, albeit not favored by naturalness principle, is to simply complete to a linear sigma model above $10 \mathrm{TeV}$.
} 
II. $S U(5)_{l} \times S O(5)_{r} / S O(5)_{v}$

The non-linear sigma model here is based on the symmetry breaking pattern $S U(5)_{r} \times$ $S O(5)_{r} / S O(5)_{v}$, where $S O(5)_{v}=S O(5)_{l+r}$. Using the same basis as in the littlest Higgs model in Ref. [12], we write the generators in terms of $X_{l}^{a}, T_{l}^{a}$, and $T_{r}^{a}$, where $X_{l}^{a}$ sit in the coset space $S U(5)_{l} / S O(5)_{l}$ and $T_{i}, i=l, r$, sit in the $S O(5)_{i}, i=l, r$, respectively. The Lie algebra of the full group looks schematically like

$$
\left[T_{l}^{a}, X_{l}^{b}\right] \sim X_{l}^{c}, \quad\left[X_{l}^{a}, X_{l}^{b}\right] \sim T_{l}^{c}, \quad\left[T_{i}^{a}, T_{i}^{b}\right] \sim T_{i}^{c}, \quad i=l, r .
$$

All other commutators are zero. The broken generators are $X_{l}^{a}$ and $X_{t} \equiv T_{l}^{a}-T_{r}^{a}$ with the corresponding Goldstone bosons $\Pi_{l}=\pi_{l}^{a} X_{l}^{a}$ and $\Pi_{t}=\pi_{t}^{a} X_{t}^{a}$, which are parametrized as

$$
\xi_{o}=e^{i \Pi_{l} / f} e^{i \Pi_{t} / f} \equiv \xi_{l} \xi_{t} \rightarrow g_{l} g_{r} \xi U
$$

where $g_{l}, g_{r}$, and $U$ belongs to $S U(5)_{l}, S O(5)_{r}$, and $S O(5)_{v}$, respectively. Moreover, $U$ furnishes a non-linear representation of $S U(5)_{l} \times S O(5)_{r}$, as was shown in CCWZ. Strictly speaking the coset space $S U(5)_{r} \times S O(5)_{r} / S O(5)_{v}$ is not a symmetric space, however, there is a $Z_{2}$ symmetry in Eq. (11) which can serve as the ascendent of $T$-parity:

$$
\mathcal{Z}_{l}: X_{l} \rightarrow-X_{l}, \quad T_{i} \rightarrow T_{i}, \quad i=l, r .
$$

Note that had we discarded the $S O(5)_{r}$ group, this would be the same $Z_{2}$ symmetry which makes it possible to impose $T$-parity on the littlest Higgs model in Ref. [30]. The action of $\mathcal{Z}_{l}$ on $\xi_{o}$ gives

$$
\mathcal{Z}_{l}\left(\xi_{o}\right)=\xi_{l}^{\dagger} \xi_{t} \rightarrow \tilde{g}_{l} g_{r} \mathcal{Z}_{l}\left(\xi_{o}\right) U
$$

where $\mathcal{Z}_{l}: g_{l}=e^{i\left(\epsilon_{l} \cdot T_{l}+\eta_{l} \cdot X_{l}\right)} \rightarrow \tilde{g}_{l}=e^{i\left(\epsilon_{l} \cdot T_{l}-\eta_{l} \cdot X_{l}\right)}$. Taking the product of $\xi_{o}$ and $\mathcal{Z}_{l}\left(\xi_{o}\right)^{\dagger}$ we deduce that

$$
\xi_{l}^{2} \rightarrow g_{l} \xi_{l}^{2} \tilde{g}_{l}^{\dagger}
$$

which then implies

$$
\xi_{l} \rightarrow g_{l} \xi_{l} U_{l}^{\dagger}=U_{l} \xi_{l} \tilde{g}_{l}^{\dagger}
$$

The element $U_{l}$ belongs to the $S O(5)_{l}$ group and furnishes a non-linear representation of $S U(5)_{l}$ as in the $T$-invariant $S U(5) / S O(5)$ model. Furthermore, given the vaccum expectation value in the littlest Higgs model

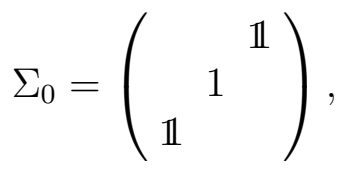

which satisfies $\Sigma_{0} T_{l} \Sigma_{0}=-T_{l}^{T}$ and $\Sigma_{0} X_{l} \Sigma_{0}=X_{l}^{T}, \tilde{g}_{l}$ has the property that $\Sigma_{0} \tilde{g}_{l}^{\dagger} \Sigma_{0}=g_{l}^{T}$. Therefore, if one defines $\Sigma_{l}=\xi_{l}^{2} \Sigma_{0}$, it would transform as

$$
\Sigma_{l} \rightarrow g_{l} \Sigma_{l} g_{l}^{T}
$$

That is, the $\Sigma_{l}$ transform just like the $\Sigma(x)$ in the littlest Higgs model and can be used to write down the kinetic term for the Goldstone bosons in $\Pi_{l}$. On the other hand, the kinetic term for $\Pi_{t}$ can be obtained using the object ${ }^{2}$

$$
\xi_{t}^{2} \rightarrow U_{l} \xi_{t}^{2} g_{r}^{\dagger}
$$

\footnotetext{
${ }^{2}$ Eq. (9) can be most easily deduced from Eq. (6) and Eq. (22), which is to be introduced later.
} 
If we ignore the generators $X_{l}^{a}$, the coset space becomes $S O(5)_{l} \times S O(5)_{r} / S O(5)$ and $\xi_{t}^{2}$ transforms in the familiar way $\xi_{t}^{2} \rightarrow g_{l} \xi_{t}^{2} g_{r}^{\dagger}$.

In the gauge sector, in the spirit of the three-site moose model in Ref. [9], three copies of $S U(2) \times U(1)$ are gauged:

$$
\begin{aligned}
& Q_{l 1}^{a}=\left(\sigma^{a} / 2 \quad\right)_{l}, \quad Y_{l 1}=\frac{1}{10} \operatorname{diag}(3,3,-2,-2,-2)_{l}, \\
& Q_{l 2}^{a}=\left(-\sigma^{a *} / 2\right)_{l}, \quad Y_{l 2}=\frac{1}{10} \operatorname{diag}(2,2,2,-3,-3)_{l}, \\
& Q_{r}^{a}=\left(\begin{array}{ll}
\sigma^{a} / 2 & \\
& -\sigma^{a *} / 2
\end{array}\right)_{r}, \quad Y_{r}=\frac{1}{2} \operatorname{diag}(1,1,0,-1,-1)_{r} .
\end{aligned}
$$

Note that $Q_{l 1}+Q_{l 2}$ and $Q_{r}$ belong to $S O(5)_{l}$ and $S O(5)_{r}$, respectively. The unbroken generators $Q^{a}$ and broken generators $\mathcal{Q}^{a}$ are thus

$$
Q=Q_{l 1}+Q_{l 2}+Q_{r} ; \quad \mathcal{Q}_{-}=Q_{l 1}-Q_{l 2} ; \quad \mathcal{Q}_{+}=Q_{l 1}+Q_{l 2}-2 Q_{r}
$$

The unbroken $S U(2) \times U(1)$ is naturally taken to be the electroweak group $S U(2)_{W} \times U(1)_{Y}$. The basis chosen in Eq. (13) is the eigenbasis of $\mathcal{Z}_{l}$ which interchanges $Q_{l 1}$ and $Q_{l 2} ; Q$ and $\mathcal{Q}_{+}$are even under $\mathcal{Z}_{l}$ whereas $\mathcal{Q}_{-}$is odd. This defines the $T$-parity for the gauge bosons, which forces the gauge couplings $g_{l 1}=g_{l 2}$. The heavy gauge bosons, $\mathcal{Q}_{+}$and $\mathcal{Q}_{-}$, have masses of the order $g f$, which is typically at around $1 \mathrm{TeV}$. The $T$-even heavy gauge bosons could in principle couple directly to the standard model matter, which is also even under $T$-parity, and contribute dangerously to the precision electroweak observables. This can be avoided by taking the gauge coupling $g_{r}$ to be somewhat strong, right below $4 \pi$, and thus raising the mass of $\mathcal{Q}_{+}$to be around $10 \mathrm{TeV}$. Then $\mathcal{Q}_{+}^{a}$ consist of mostly the gauge bosons in $Q_{r}^{a}$ and, if the standard model matter is not charged under the strongly coupled gauge group $Q_{r}^{a}$, their dangerous tree-level contributions to the precision measurements are suppressed and hence safe. The gauge sector here is completely similar to that of the three-site moose model with $T$-parity in Ref. [9].

With the gauge fields defined above and the transformation properties given in Eqs. (8) and (9), we can write down the scalar kinetic terms for the $\Pi_{l}$ and $\Pi_{t}$

$$
\mathcal{L}_{X} \supset \frac{f^{2}}{8}\left(\operatorname{Tr}\left|D_{\mu} \Sigma_{l}\right|^{2}+\operatorname{Tr}\left|D_{\mu} \xi_{t}^{2}\right|^{2}\right),
$$

where

$$
\begin{aligned}
& D \Sigma_{l}=\partial \Sigma_{l}-\sum_{j=1,2}\left\{i g_{l j} W_{l j}^{a}\left(Q_{l j}^{a} \Sigma_{l}+\Sigma_{l} Q_{l j}^{a T}\right)+i g_{l j}^{\prime} B_{l j}\left(Y_{l j} \Sigma_{l}+\Sigma_{l} Y_{l j}^{T}\right)\right\} \\
& D \xi_{t}^{2}=\partial \xi_{t}^{2}-v_{l}^{a} T_{l}^{a} \xi_{t}^{2}+\xi_{t}^{2}\left(i g_{r} W_{r}^{a} Q_{r}^{a}+i g_{r}^{\prime} B_{r} Y_{r}\right) .
\end{aligned}
$$

The object $v_{l \mu}^{a} T_{l}^{a}$ in $D_{\mu} \xi_{t}^{2}$ can be written compactly as $v_{l \mu}^{a} T_{l}^{a}=\frac{1}{2}\left(\xi_{l}^{\dagger} D_{\mu} \xi_{l}+\xi_{l} D_{\mu} \xi_{l}^{\dagger}\right)$ which is defined in Ref. [30] and shown to transform under the $U_{l}$ rotation like a gauge field: $v_{l \mu}^{a} T_{l}^{a} \rightarrow U_{l}\left(v_{l \mu}^{a} T_{l}^{a}\right) U_{l}^{\dagger}+U_{l} \partial_{\mu} U_{l}^{\dagger}$. In fact, the detailed form of $D_{\mu} \xi_{t}^{2}$ need not concern us here since all the uneaten Goldstone bosons residing in $\xi_{t}^{2}$ will be lifted to be massive at around $10 \mathrm{TeV}$ and beyond the reach of precision measurements. 
There are 14 Goldstone bosons in $\Pi_{l}$ and 10 Goldstone bosons in $\Pi_{t}$. Their quantum numbers under the $S U(2)_{W} \times U(1)_{Y}$ are

$$
\begin{aligned}
& \Pi_{l}: 1_{0} \oplus 2_{ \pm 1 / 2} \oplus 3_{0} \oplus 3_{ \pm 1}, \\
& \Pi_{t}: 1_{0} \oplus 1_{ \pm 1} \oplus 2_{ \pm 1 / 2} \oplus 3_{0} .
\end{aligned}
$$

The action of $T$-parity on the scalars is defined as

$$
T: \Pi_{l} \rightarrow-\Omega \Pi_{l} \Omega, \quad \Pi_{t} \rightarrow \Omega \Pi_{t} \Omega,
$$

where $\Omega=\operatorname{diag}(1,1,-1,1,1)$ ensures that the Higgs doublet, which is taken to be the electroweak doublet residing in $\Pi_{l}$, is even under $T$-parity. Among the scalars in Eq. (17), two copies of $1_{0} \oplus 3_{0}$, one from $\Pi_{l}$ and the other from $\Pi_{t}$, are eaten by the heavy gauge bosons through Higgs mechanism. The complex triplet in $\Pi_{l}$ become massive through a potential radiatively generated at one loop due to the gauge covariant derivative in Eq. (15):

$$
\sum_{j=1,2}\left\{c_{l} g_{l j}^{2} f^{4} \sum_{a} \operatorname{Tr}\left[\left(Q_{l j}^{a} \Sigma_{l}\right)\left(Q_{l j}^{a} \Sigma_{l}\right)^{*}\right]+c_{l} g_{l j}^{\prime 2} f^{4} \operatorname{Tr}\left[\left(Y_{l j} \Sigma_{l}\right)\left(Y_{l j} \Sigma_{l}\right)^{*}\right]\right\},
$$

The remaining scalars are one complex doublet and one complex singlet, both living in $\Pi_{t}$. They can be given a mass through the plaquette operator

$$
\epsilon_{1} f^{4} \operatorname{Tr}\left[\Omega^{\prime} \xi_{t}^{2} \Omega^{\prime}\left(\xi_{t}^{\dagger}\right)^{2}\right]
$$

where $\Omega^{\prime}=\operatorname{diag}(1,1,-1,-1,-1)$. This plaquette operator is invariant under gauge transformations $^{3}$ as well as the $T$-parity. If $\epsilon_{1}$ is of order unity, the scalars are massive at around $1 \mathrm{TeV}$. However, we can further raise the scalar masses in $\xi_{t}$ to $10 \mathrm{TeV}$ by making the plaquette strongly coupled, $\epsilon_{1} \sim 4 \pi$. In so doing, the gauge and scalar sectors below $10 \mathrm{TeV}$ is the same as in the littlest Higgs model, which has one set of $S U(2) \times U(1)$ gauge bosons and one complex triplet scalar, all are massive at around $1 \mathrm{TeV}$, as well as one light Higgs doublet at the order $100 \mathrm{GeV}$.

The structure of this model in the $S U(5)_{l}$ sector is very similar to the $S U(5) / S O(5)$ littlest Higgs model. The Higgs doublet and the triplet scalars are both from the $S U(5)_{l}$ sector

$$
\Pi_{l}=\left(\begin{array}{ccc} 
& \frac{H}{\sqrt{2}} & \phi \\
\frac{H^{\dagger}}{\sqrt{2}} & & \frac{H^{T}}{\sqrt{2}} \\
\phi^{\dagger} & \frac{H^{*}}{\sqrt{2}} &
\end{array}\right) .
$$

So is the $T$-odd heavy gauge bosons, $\mathcal{Q}_{-}=Q_{l 1}-Q_{l 2}$. All the scalars and gauge bosons associated with $S U(5)_{r}$ are made heavy at around $10 \mathrm{TeV}$. The lightness of the Higgs doublet originates from the little Higgs mechanism working in the $S U(5)_{l}$ sector, in exactly the same way as the littlest Higgs model. The gauge interaction in $\operatorname{Tr}\left|D_{\mu} \Sigma_{l}\right|^{2}$ in Eq. (14) is identical to that of the $\Sigma(x)$ in the littlest Higgs and radiatively generates a potential, Eq. (19), giving the triplet mass, as well as the Higgs quartic coupling once the triplet is integrated out [12]. The one loop quadratic contribution to the Higgs mass-squared coming from the

\footnotetext{
${ }^{3}$ For the purpose of verifying gauge invariance, the symmetry breaking pattern can be thought of as $S U(2)_{l 1} \times S U(2)_{l 2} \times S U(2)_{r} / S U(2)_{v}$. Then $\Omega^{\prime}$ commutes with $U_{l}$ when restricted to the gauge groups.
} 
Higgs quartic coupling is canceled by contributions from the triplet scalar $\phi$ in Eq. (21); all other scalars are not involved and can be as heavy as $10 \mathrm{TeV}$, and hence decouple from the spectrum.

In the fermionic sector, the $T$-parity, which interchanges the two gauge groups within $S U(5)_{l}$, forces identical fermion contents charged under $S U(2)_{l 1}$ and $S U(2)_{l 2}$. A mirror fermion, charged under $S U(2)_{r}$ which is neutral under $T$-parity, can be introduced to marry one linear combination of the fermions charged under $S U(2)_{l 1}$ and $S U(2)_{l 2}$. The remaining massless linear combination will be taken to be the standard model doublet fermion, which does not carry any charged under the strongly coupled gauge group $S U(2)_{r}$. Again this is reminiscent of the fermionic sector in the three-site moose model in Ref. 9]. In order to marry fermions charged under $S U(5)_{l}$ and $S O(5)_{r}$, we need a function of Goldstone bosons which transforms as bi-fundamental under both groups and then introduce a Yukawa type interaction. The function with the desired property is

$$
X_{m} \equiv \xi_{l} \xi_{t}^{2} \rightarrow g_{l} X_{m} g_{r}^{\dagger}
$$

which can be proven using CCWZ, who showed when one can and how to construct a function of only Goldstone bosons with a desired transformation property. In the linear sigma model $X_{m}$ can be obtained from parametrizing the Goldston bosons over the vaccum expectation value of a scalar $\Phi \rightarrow g_{l} \Phi g_{r}^{\dagger}$. A subtlety arises here because the physical Higgs doublet is contained in $X_{m}$, which forces the mirror fermions to come in complete $S O(5)_{r}$ multiplet; otherwise a quadratic divergence to the Higgs mass would be induced through the Yukawa-type interaction. Therefore for each electroweak doublet in the standard model, one introduces the following fermions:

$$
\Psi_{l 1}=\left(\begin{array}{c}
\psi_{l 1} \\
0 \\
0
\end{array}\right) ; \quad \Psi_{l 2}=\left(\begin{array}{c}
0 \\
0 \\
\psi_{l 2}
\end{array}\right) ; \quad \Psi_{r}^{c}=\left(\begin{array}{c}
\psi_{r}^{c} \\
\chi_{r}^{c} \\
\widetilde{\psi}_{r}^{c}
\end{array}\right) ; \quad \Psi_{r}=\left(\begin{array}{c}
0 \\
\chi_{r} \\
\tilde{\psi}_{r}
\end{array}\right)
$$

where $\Psi_{l 1}$ and $\Psi_{l 2}$ transform only under $S U(5)_{l}$, and $\Psi_{r}^{c}$ and $\Psi_{r}$ transform only under $S O(5)_{r}$. Note that $\Psi_{l 1}$ and $\Psi_{l 2}$ have the same charge under the diagonal gauge group $S U(2)_{W} \times U(1)_{Y}$. Under $T$-parity,

$$
T: \Psi_{l 1} \rightarrow-\Sigma_{0} \Psi_{l 2}, \quad \Psi_{r}^{c} \rightarrow-\Psi_{r}^{c}, \quad \Psi_{r} \rightarrow-\Psi_{r} .
$$

The minus sign in the above serves to make the heavy fermions odd under $T$-parity. Then the masses of the heavy, $T$-odd fermions could come from the following interactions

$$
\kappa_{1} f\left(\bar{\Psi}_{l 1} X_{m} \Psi_{r}^{c}+\bar{\Psi}_{l 2} \Sigma_{0} \tilde{X}_{m} \Psi_{r}^{c}\right)+\kappa_{2} \Psi_{r}^{T} \Psi_{r}^{c}+\text { h.c. }
$$

where $\tilde{X}_{m}=\Omega \xi_{l}^{\dagger} \xi_{t}^{2} \Omega$ is the image of $X_{m}$ under $T$-parity. Eq. (25) gives a mass to the Dirac pair $\left(\psi_{l 1}+\psi_{l 2}, \psi_{r}^{c}\right),\left(\chi_{r}, \chi_{r}^{c}\right)$, and $\left(\tilde{\psi}_{r}, \tilde{\psi}_{r}^{c}\right)$, which are odd under the $T$-parity defined in Eq. (24). The standard model doublet is taken to be the massless, $T$-even combination $\psi_{s m} \equiv\left(\psi_{l 1}-\psi_{l 2}\right) / \sqrt{2}$. We will see in the next section that for $H_{r}=S U(5)$ less number of fermions is required and only $\psi_{l 1}, \psi_{l 2}$, and $\psi_{r}^{c}$ are necessary.

For Yukawa coupling, it suffices to focus on the top sector which generates a large contribution to the Higgs mass. We write the third generation quark doublet as $q^{T}=(b t)=$ $\left(q_{l 1}^{T}-q_{l 2}^{T}\right) / \sqrt{2}$, where $q_{l j}^{T}=\left(b_{l j} t_{l j}\right)$, for $j=1,2$. One also needs to introduce four colored 
weak singlet fermions $t_{l 1}^{\prime}, t_{l 2}^{\prime}, t_{r}^{\prime c}$, and $t^{\prime c}$, and group them with the third generation quark in the following fashion

$$
Q_{l 1}=\left(\begin{array}{c}
q_{l 1} \\
t_{l 1}^{\prime} \\
0
\end{array}\right), \quad Q_{l 2}=\left(\begin{array}{c}
0 \\
t_{l 2}^{\prime} \\
q_{l 2}
\end{array}\right) .
$$

The top Yukawa coupling is contained in the following interaction, summing $i, j, k$ over 1 , 2,3 and $x, y$ over 4,5 ,

$$
\frac{1}{2 \sqrt{2}} \lambda_{1} f \epsilon_{i j k} \epsilon_{x y}\left[\left(Q_{l 1}\right)_{i}\left(\Sigma_{l}\right)_{j x}\left(\Sigma_{l}\right)_{k y}-\left(\Sigma_{0} Q_{l 2}\right)_{i}\left(\tilde{\Sigma}_{l}\right)_{j x}\left(\tilde{\Sigma}_{l}\right)_{k y}\right] u_{3}^{\prime c}+\lambda_{2} f t_{+}^{\prime} t^{\prime c}+\lambda_{3} f t_{-}^{\prime} t_{r}^{c},
$$

where $t_{ \pm}^{\prime}=\left(t_{l 1}^{\prime} \mp t_{l 2}^{\prime}\right) / \sqrt{2}$. The top Yukawa coupling here is the $T$-symmetried version of the top Yukawa coupling in Ref. [12]. The image of $\Sigma_{l}$ under the $T$-parity, defined as $\tilde{\Sigma}_{l}$ in the above, is $\Omega\left(\xi_{l}^{\dagger}\right)^{2} \Sigma_{0} \Omega$. The singlet fermions $t_{-}^{\prime}$ and $t_{r}^{c}$ are defined to be odd under $T$-parity, whereas $t_{+}^{\prime}, t^{\prime c}$, and $u_{3}^{\prime c}$ are all $T$-even. At leading order in the Higgs particle, Eq. (27) gives

$$
\lambda_{1}\left(\sqrt{2} H q+f t_{+}^{\prime}\right) u_{3}^{\prime c}+\lambda_{2} f t_{+}^{\prime} t^{\prime c}+\lambda_{3} f t_{-}^{\prime} t_{r}^{c} .
$$

Therefore $t_{-}^{\prime}$ marries $t_{r}^{c}$ to become massive, and similarly for $t_{+}^{\prime}$ and $\lambda_{1} u_{3}^{\prime c}+\lambda_{2} t^{\prime c}$. The remaining massless combination $u_{3}^{c}=\left(\lambda_{2} u_{3}^{\prime c}-\lambda_{1} t^{\prime c}\right) / \sqrt{\lambda_{1}^{2}+\lambda_{2}^{2}}$ has the desired Yukawa coupling to $q$ with the strength $\lambda_{t}=\sqrt{2} \lambda_{1} \lambda_{2} / \sqrt{\lambda_{1}^{2}+\lambda_{2}^{2}}$.

III. $S U(5)_{l} \times S U(5)_{r} / S O(5)_{v}$

In this section we enlarge $H_{r}$ to $S U(5)$ and show that less number of fermions are needed for the construction. Similar to the case with $H_{r}=S O(5)$, all the extra Goldstone bosons can be made heavy at $10 \mathrm{TeV}$ and integrated out of the spectrum. Written in terms of $T_{i}^{a} \in S O(5)_{i}$ and $X_{i}^{a} \in S U(5)_{i} / S O(5)_{i}$, the Lie algebra of the full symmetry group is, again schematically,

$$
\left[T_{i}^{a}, T_{i}^{b}\right] \sim T_{i}^{c}, \quad\left[T_{i}^{a}, X_{i}^{b}\right] \sim X_{i}^{c}, \quad\left[X_{i}^{a}, X_{i}^{b}\right] \sim T_{i}^{c}, \quad i=l, r
$$

The broken generators in this case are $X_{l}^{a}, X_{r}^{a}$, and $X_{t}^{a} \equiv T_{l}^{a}-T_{r}^{a}$. The Goldstone bosons $\Pi_{l}=\pi_{l}^{a} X_{l}^{a}, \Pi_{r}=\pi_{r}^{a} X_{r}^{a}$, and $\Pi_{t}=\pi_{t}^{a} X_{t}^{a}$ can be parametrized as

$$
\xi_{u}=e^{i \Pi_{l} / f} e^{i \Pi_{r} / f} e^{i \Pi_{t} / f} \equiv \xi_{l} \xi_{r} \xi_{t} \rightarrow g_{l} g_{r} \xi U,
$$

where $g_{l}, g_{r}$, and $U$ belongs to $S U(5)_{l}, S U(5)_{r}$, and $S O(5)_{v}$, respectively. Note that $\left[X_{l}^{a}, X_{r}^{b}\right]=0$ so $\xi_{l} \xi_{r}=\xi_{r} \xi_{l}$. Eq. (29) has several useful $Z_{2}$ symmetries:

$$
\mathcal{Z}_{l}: X_{l} \rightarrow-X_{l} ; \quad \mathcal{Z}_{r}: X_{r} \rightarrow-X_{r} ; \quad \mathcal{Z}_{t}: X_{l} \rightarrow X_{r}, T_{l} \rightarrow T_{r}
$$

Taking the product of $\xi_{u}$ and $\mathcal{Z}_{i}\left(\xi_{u}\right)^{\dagger}$ and define $\Sigma_{i}=\xi_{i}^{2} \Sigma_{0}, i=l, r$, we deduce that

$$
\Sigma_{i}^{2} \rightarrow g_{i} \Sigma_{i}^{2} g_{i}^{T}, \quad i=l, r ; \quad \Sigma_{t} \equiv \xi_{l} \xi_{r}\left(\xi_{t}\right)^{2} \xi_{r}^{\dagger} \xi_{l}^{\dagger} \rightarrow g_{l} \Sigma_{t} g_{r}^{\dagger},
$$

where in obtaining the transformation property for $\Sigma_{t}$ we have focused on the special case when the group action in Eq. (30) is restricted to $g_{l}$. We can use the $\Sigma$ s to write down the kinetic terms for the Goldstone bosons:

$$
\mathcal{L}_{X} \supset \frac{f^{2}}{8}\left(\operatorname{Tr}\left|D_{\mu} \Sigma_{l}\right|^{2}+\operatorname{Tr}\left|D_{\mu} \Sigma_{r}\right|^{2}+\operatorname{Tr}\left|D_{\mu} \Sigma_{t}\right|^{2}\right)
$$


where $D \Sigma_{l}$ is the same as in Eq. (15) and

$$
\begin{aligned}
& D \Sigma_{r}=\partial \Sigma_{r}-\left\{i g_{r} W_{r}^{a}\left(Q_{r}^{a} \Sigma_{r}+\Sigma_{r} Q_{r}^{a T}\right)+i g_{r}^{\prime} B_{r}\left(Y_{r} \Sigma_{r}+\Sigma_{r} Y_{r}^{T}\right)\right\} \\
& D \Sigma_{t}=\partial \Sigma_{t}+\Sigma_{t}\left(i g_{r} W_{r}^{a} Q_{r}^{a}+i g_{r}^{\prime} B_{r} Y_{r}\right)-\sum_{j=1,2}\left(i g_{l j} W_{l j}^{a} Q_{l j}^{a}+i g_{l j}^{\prime} B_{l j} Y_{l j}\right) \Sigma_{t} .
\end{aligned}
$$

In the above we have gauged the same generators as in the $H_{r}=S O(5)$ case. Even though $\Pi_{l}$ and $\Pi_{r}$ are contained in $\Sigma_{t}=\xi_{l} \xi_{r}\left(\xi_{t}\right)^{2} \xi_{r}^{\dagger} \xi_{l}^{\dagger}$, the interaction $\operatorname{Tr}\left|D_{\mu} \Sigma_{t}\right|^{2}$ does not give rise to extra kinetic terms for $\Pi_{l}$ and $\Pi_{r}$.

There are $14+14+10$ Goldstone bosons in total with the electroweak quantum numbers

$$
\begin{aligned}
& \Pi_{l}: 1_{0} \oplus 2_{ \pm 1 / 2} \oplus 3_{0} \oplus 3_{ \pm 1}, \\
& \Pi_{r}: 1_{0} \oplus 2_{ \pm 1 / 2} \oplus 3_{0} \oplus 3_{ \pm 1}, \\
& \Pi_{t}: 1_{0} \oplus 1_{ \pm 1} \oplus 2_{ \pm 1 / 2} \oplus 3_{0} .
\end{aligned}
$$

That is, there are three real singlets, one complex singlet, three complex doublet, three real triplets, and two complex triplets. The action of $T$-parity, which descends from the $Z_{2}$ symmetry $\mathcal{Z}_{l}$, on the scalars is

$$
T: \Pi_{l} \rightarrow-\Omega_{t} \Pi_{l} \Omega_{t}, \quad \Pi_{r} \rightarrow \Omega_{t} \Pi_{r} \Omega_{t}, \quad \Pi_{t} \rightarrow \Omega_{t} \Pi_{t} \Omega_{t} .
$$

Among the scalars in Eq. (37), two copies of $1_{0} \oplus 3_{0}$ in $\Pi_{l}$ and $\Pi_{t}$ are eaten by the heavy gauge bosons as in the $H_{r}=S O(5)$ case. The two complex triplets in $\Pi_{l}$ and $\Pi_{r}$, as well as the complex doublet in $\Pi_{r}$, become massive through a potential radiatively generated at one loop by the gauge interactions in Eq. (333):

$$
\begin{array}{r}
\sum_{j=1,2}\left\{c_{l} g_{l j}^{2} f^{4} \sum_{a} \operatorname{Tr}\left[\left(Q_{l j}^{a} \Sigma_{l}\right)\left(Q_{l j}^{a} \Sigma_{l}\right)^{*}\right]+c_{l} g_{l j}^{\prime 2} f^{4} \operatorname{Tr}\left[\left(Y_{l j} \Sigma_{l}\right)\left(Y_{l j} \Sigma_{l}\right)^{*}\right]\right\} \\
+c_{r} g_{r}^{2} f^{4} \sum_{b} \operatorname{Tr}\left[\left(Q_{r}^{b} \Sigma_{r}\right)\left(Q_{r}^{b} \Sigma_{r}\right)^{*}\right]+c_{r} g_{r}^{\prime 2} f^{4} \operatorname{Tr}\left[\left(Y_{r} \Sigma_{r}\right)\left(Y_{r} \Sigma_{r}\right)^{*}\right]
\end{array}
$$

where the first line is generated by gauge groups sitting in $S U(5)_{l}$ and gives a mass of order $g_{l} f \sim 1 \mathrm{TeV}$ to the triplet in $\Pi_{l}$, while the second line, generated by the gauge groups in $S U(5)_{r}$, gives masses of order $g_{r} f \sim 10 \mathrm{TeV}$ to every scalar in $\Pi_{r}$ except the singlet. The scalars in $\Pi_{r}$ are at around $10 \mathrm{TeV}$ because the gauge coupling is strong, $g_{r} \sim 4 \pi$. The remaining scalars, which do not obtain a mass through Eq. (38), are two complex doublets, residing in $\Pi_{l}$ and $\Pi_{t}$ respectively, one complex singlet from $\Pi_{t}$, and one real singlet in $\Pi_{r}$. To ensure that there is only one light electroweak doublet, which is the Higgs, we can put in the following plaquette operators

$$
\mathcal{L}_{X} \supset \epsilon_{1} f^{4} \operatorname{Tr}\left(\Omega^{\prime} \Sigma_{t} \Omega^{\prime} \Sigma_{t}^{\dagger}\right)+\epsilon_{2} f^{4} \operatorname{Tr}\left(\xi_{r}^{2}+\Omega \xi_{r}^{2} \Omega\right) .
$$

If $\epsilon_{i} \sim 4 \pi, i=1,2$, all the scalars, except for the doublet and the triplet in $\Pi_{l}$, are massive at $10 \mathrm{TeV}$ and we have the same gauge and scalar sectors below $10 \mathrm{TeV}$ as in the littlest Higgs model.

In the fermionic sector, again we need to introduce identical fermion contents charged under $S U(2)_{l 1}$ and $S U(2)_{l 2}$, and mirror fermions charged under $S U(2)_{r}$. We will use $\Sigma_{t}$ 
to write down a Yukawa-type interaction to give the TeV fermion a mass. Here the mirror fermions do not have to fill a complete multiplet of the $S U(5)_{r}$ because the induced quadratic divergence gives mass to only the doublet in $\Pi_{t}$, but not the physical Higgs in $\Pi_{l}$ :

$$
\Psi_{l 1}=\left(\begin{array}{c}
\psi_{l 1} \\
0 \\
0
\end{array}\right) ; \quad \Psi_{l 2}=\left(\begin{array}{c}
0 \\
0 \\
\psi_{l 2}
\end{array}\right) ; \quad \Psi_{r}^{c}=\left(\begin{array}{c}
\psi_{r}^{c} \\
0 \\
0
\end{array}\right)
$$

where $\Psi_{l 1}$ and $\Psi_{l 2}$ transform only under $S U(5)_{l}$ and $\Psi_{r}^{c}$ transforms only under $S U(5)_{r}$. Under $T$-parity,

$$
T: \Psi_{l 1} \rightarrow-\Sigma_{0} \Psi_{l 2}, \quad \Psi_{r}^{c} \rightarrow-\Psi_{r}^{c} .
$$

Then the masses of the heavy, $T$-odd fermions could come from the following interactions

$$
\kappa f\left(\bar{\Psi}_{l 1} \Sigma_{t} \Psi_{r}^{c}+\bar{\Psi}_{l 2} \Sigma_{0} \tilde{\Sigma}_{t} \Psi_{r}^{c}\right)
$$

where $\tilde{\Sigma}_{t}=\Omega\left(\xi_{l}^{\dagger} \xi_{r} \xi_{t}^{2} \xi_{r}^{\dagger} \xi_{l}\right) \Omega$. At one loop Eq. (42) generates a plaquette operators similar to the $\epsilon_{1}$ term in Eq. (39), which gives the doublet and singlet scalars in $\Pi_{t}$ masses in the TeV range. The top Yukawa coupling can be written down in a fashion identical to Eq. (27) by introducing additional singlet fermions. In the end there are fewer number of heavy fermions at $1 \mathrm{TeV}$ than in the $H_{r}=S O(5)$ case, while the numbers of gauge and scalar particles below $10 \mathrm{TeV}$ in both cases are identical, which are the same as in the littlest Higgs model.

\section{DISCUSSIONS}

So far we have constructed extensions of the littlest Higgs model with $T$-parity. In all cases encountered so far, whether we choose to utilize CCWZ or not, the spectrum in the gauge and scalar sectors can be the same as in the original littlest Higgs model; extra gauge bosons and scalar particles can be lifted to be heavy at around the cutoff scale and integrated out. One can ask the question whether it is possible to lift the mass of the mirror fermions to $10 \mathrm{TeV}$ as well and thus obtain a spectrum identical to the littlest Higgs model. It turns out not possible to do so because the Yukawa-type interactions giving masses to the mirror fermions, Eqs. (25) and (42), contain vertices with one standard model fermion, one mirror fermion, and a Goldstone boson which look like $\kappa f \bar{\psi}_{s m} \pi^{a} \psi_{r}^{c}$. Such a vertex would, at one loop through the box diagram, give a finite contribution to the four-fermion operator $c_{f}\left(\bar{\psi}_{s m} \bar{\sigma}_{\mu} \psi_{s m}\right)\left(\bar{\psi}_{s m} \bar{\sigma}^{\mu} \psi_{s m}\right)$ with

$$
c_{f} \sim \frac{1}{16 \pi^{2}}(\kappa f)^{4}\left(\frac{1}{f^{2}}\right)^{2} \frac{1}{(\kappa f)^{2}}=\frac{1}{16 \pi^{2}} \frac{\kappa^{2}}{f^{2}},
$$

where $\left(1 / f^{2}\right)^{2}$ comes from the two Goldstone boson propagators and $1 /(\kappa f)^{2}$ from the mass term in the fermion propagators. The size of such four-fermion operators is severely constrained by various experiments to be $c_{f} \leq 1 /(5-15 \mathrm{TeV})^{2}$ [34]. If we take $f$ to be its natural size $\sim 1 \mathrm{TeV}$, the mass of the mirror fermion is then constrained to be $\kappa f \leq 0.8 \mathrm{TeV}$. That is the heavy fermions need to be slightly lighter than $1 \mathrm{TeV}$ to cutoff the dangerous contribution to the four-fermion operators.

Such constraints from the four-fermion operators are in fact not considered in the original construction of $T$-invariant models using CCWZ in Ref. [30]. It turns out that, because 
the standard model fermions there have the kinetic term $\bar{\psi}\left(\xi^{\dagger} D_{\mu} \xi+\xi D_{\mu} \xi^{\dagger}\right) \psi$, four-fermion operators are generated with unsuppressed coefficients $1 / f^{2}$, which would force $f$ to be in the order of $10 \mathrm{TeV}$ and introduce fine-tuning to the Higgs mass. ${ }^{4}$ Nevertheless, the model with $H_{r}=S O(5)$ in Section II inspires an alternative construction using CCWZ, without extending the global/gauge symmetry group, which is safe from large four-fermion operators. The construction proceeds as follows. In the $T$-invariant $S U(5) / S O(5)$ model in Ref. [30] all the fermions are assigned to transform under the unbroken $S O(5)$ according to the prescription of CCWZ:

$$
\psi=\left(\begin{array}{c}
\psi_{1} \\
\chi \\
\psi_{2}
\end{array}\right) \rightarrow U \psi
$$

where $U$ belongs to the unbroken $S O(5)$ and non-linearly realizes the full $S U(5)$ group. This assignment of fermion representation has the advantage of getting rid of all the tree-level contributions to the electroweak observables from new particles at $1 \mathrm{TeV}$. However, it also results in the particular kinetic term giving rise to large four-fermion operators. The remedy is to follow the idea of introducing mirror fermions whose lightness serves to cutoff the size of the four-fermion operators. That is, we will introduce two doublets $\varphi_{1}$ and $\varphi_{2}$ transforming linearly under $S U(2)_{1}$ and $S U(2)_{2}$ respectively. These two doublets are mapped into each other under $T$-parity which interchanges $S U(2)_{1}$ and $S U(2)_{2}$. The mirror fermions, which need to be neutral under $T$-parity, will be assigned to a complete multiplet of the unbroken $S O(5)$ and transform non-linearly under $S U(5)$. More specifically,

$$
\Psi_{1}=\left(\begin{array}{c}
\varphi_{1} \\
0 \\
0
\end{array}\right) \rightarrow g_{u} \Psi_{1} ; \quad \Psi_{2}=\left(\begin{array}{c}
0 \\
0 \\
\varphi_{2}
\end{array}\right) \rightarrow g_{u} \Psi_{2} ; \quad \Psi^{c}=\left(\begin{array}{c}
\varphi^{c} \\
\chi^{c} \\
\tilde{\varphi}^{c}
\end{array}\right) \rightarrow U \Psi^{c}
$$

where $g_{u}$ is the $S U(5)$ rotation. The mirror fermion $\Psi^{c}$ needs to be in complete $S O(5)$ multiplet; otherwise a two-loop quartic divergence would be generated [30] and contribute dangerously to the Higgs mass. Again $T$-parity maps $\Psi_{1} \rightarrow-\Sigma_{0} \Psi_{2}$ and $\Psi^{c} \rightarrow-\Psi^{c}$. A Yukawa-type interaction which gives the mirror fermion a mass is

$$
\kappa f\left(\bar{\Psi}_{1} \xi \Psi^{c}+\bar{\Psi}_{2} \Sigma_{0} \Omega \xi^{\dagger} \Omega \Psi^{c}\right),
$$

where $\xi=e^{i \Pi / f} \rightarrow g_{u} \xi U^{\dagger}$. In this way the standard model fermion $\varphi_{s m}=\left(\varphi_{1}-\varphi_{2}\right) / \sqrt{2}$ has the normal kinetic term $\bar{\varphi}_{s m} D_{\mu} \varphi_{s m}$, whereas the mirror fermion $\Psi^{c}$ has the CCWZ kinetic term $\bar{\Psi}_{c}\left(\xi^{\dagger} D_{\mu} \xi+\xi D_{\mu} \xi^{\dagger}\right) \Psi_{c}$, which results in four-fermion operators consist of the $\mathrm{TeV}$ fermions, but not the standard model fermions. In order to give masses to $\chi^{c}$ and $\tilde{\varphi}^{c}$, we also need to introduce $\chi$ and $\tilde{\varphi}$ which can sit in a complete spinor representation of $S O(5)$ along with another singlet $\tilde{\chi}$, as discussed in Ref. [30]. The Yukawa coupling for the top quark can be written down in a way completely similar to Eq. (27).

The extended models with $T$-parity discussed in Sections II and III, as well as the modified CCWZ construction mentioned in the previous paragraph, solve the little hierarchy problem naturally while at the same time are consistent with data from precision measurements. After eliminating the constraint from the four-fermion operators, the strongest constraint on the $T$-invariant models comes from the correction to the $\rho$ parameter from the $T$-odd

\footnotetext{
${ }^{4}$ Correspondences with R. Rattazzi and R. Barbieri on this subject are gratefully acknowledged.
} 
gauge bosons, which only restricts the symmetry breaking scale $f$ to be larger than $450 \mathrm{GeV}$ 30]. The introduction of $T$-parity not only eliminates all the tree-level corrections to the precision observables from new particles responsible for canceling the quadratic divergence of the Higgs mass, but also adds a bonus of predicting a weakly interacting massive particle, the lightest T-odd particle (LTP), which is stable and serves as a dark matter candidate. The $T$-invariant extensions of the littlest Higgs model, whether using CCWZ or not, all share very similar phenomenology in that the $T$-odd particles need to be pair-produced. Moreover, the LTP is most likely to be the $B^{\prime}$ gauge boson, which is lighter than other $T$-odd particles because of the small $U(1)$ gauge coupling and the large normalization factor in the hypercharge assignment. Much of the discussion on the low energy phenomenology runs parallel to those in Ref. [30], since the spectra below $10 \mathrm{TeV}$ are very similar, and will not be repeated.

\section{Acknowledgments}

Numerous discussions with Hsin-Chia Cheng are gratefully acknowledged, who collaborated at various stages of this project and shared many important insights. Helpful conversations with Nima Arkani-Hamed and Jesse Thaler are also acknowledged. This work is supported in part by the National Science Foundation under grant PHY-0244821.

[1] S. Weinberg, "Phenomenological Lagrangians," PhysicaA 96, 327 (1979).

[2] W. Buchmuller and D. Wyler, "Effective Lagrangian Analysis Of New Interactions And Flavor Conservation," Nucl. Phys. B 268, 621 (1986).

[3] For a recent analysis, see R. Barbieri and A. Strumia, "What is the limit on the Higgs mass?," Phys. Lett. B 462, 144 (1999) arXiv:hep-ph/9905281.

[4] S. Weinberg, Phys. Rev. Lett. 59, 2607 (1987).

[5] N. Arkani-Hamed and S. Dimopoulos, arXiv:hep-th/0405159

[6] H. Murayama, "Flavor in supersymmetry," Prepared for Theoretical Advanced Study Institute in Elementary Particle Physics (TASI 2000): Flavor Physics for the Millennium, Boulder, Colorado, 4-30 Jun 2000

[7] N. Arkani-Hamed, talk at the CERN Conference: Strings at CERN (July 5 - 6, 2004), http://wwwth.cern.ch/strings@cern/talks/arkani.pdf

[8] J. Wudka, arXiv:hep-ph/0307339.

[9] H.-C. Cheng and I. Low, "TeV symmetry and the little hierarchy problem," JHEP 0309, 051 (2003) arXiv:hep-ph/0308199.

[10] N. Arkani-Hamed, A. G. Cohen and H. Georgi, "Electroweak symmetry breaking from dimensional deconstruction," Phys. Lett. B 513, 232 (2001) hep-ph/0105239.

[11] N. Arkani-Hamed, A. G. Cohen, E. Katz, A. E. Nelson, T. Gregoire and J. G. Wacker, "The minimal moose for a little Higgs," JHEP 0208, 021 (2002) arXiv:hep-ph/0206020.

[12] N. Arkani-Hamed, A. G. Cohen, E. Katz and A. E. Nelson, "The littlest Higgs," JHEP 0207, 034 (2002) arXiv:hep-ph/0206021.

[13] I. Low, W. Skiba and D. Smith, "Little Higgses from an antisymmetric condensate," Phys. Rev. D 66, 072001 (2002) arXiv:hep-ph/0207243.

[14] D. E. Kaplan and M. Schmaltz, "The little Higgs from a simple group," arXiv:hep-ph/0302049. 
[15] S. Chang and J. G. Wacker, "Little Higgs and custodial SU(2)," arXiv:hep-ph/0303001.

[16] S. Chang, "A 'littlest Higgs' model with custodial SU(2) symmetry," arXiv:hep-ph/0306034.

[17] W. Skiba and J. Terning, "A simple model of two little Higgses," arXiv:hep-ph/0305302.

[18] M. Schmaltz, arXiv:hep-ph/0407143.

[19] E. Katz, J. y. Lee, A. E. Nelson and D. G. E. Walker, arXiv:hep-ph/0312287.

[20] D. E. Kaplan, M. Schmaltz and W. Skiba, arXiv:hep-ph/0405257.

[21] N. Arkani-Hamed, A. G. Cohen, T. Gregoire and J. G. Wacker, "Phenomenology of electroweak symmetry breaking from theory space," JHEP 0208, 020 (2002) arXiv:hep-ph/0202089.

[22] J. L. Hewett, F. J. Petriello and T. G. Rizzo, "Constraining the littlest Higgs," arXiv:hep-ph/0211218.

[23] C. Csaki, J. Hubisz, G. D. Kribs, P. Meade and J. Terning, "Big corrections from a little Higgs," Phys. Rev. D 67, 115002 (2003) arXiv:hep-ph/0211124.

[24] C. Csaki, J. Hubisz, G. D. Kribs, P. Meade and J. Terning, "Variations of little Higgs models and their electroweak constraints," arXiv:hep-ph/0303236.

[25] T. Gregoire, D. R. Smith and J. G. Wacker, "What precision electroweak physics says about the $\mathrm{SU}(6) / \mathrm{Sp}(6)$ little Higgs," arXiv:hep-ph/0305275.

[26] C. Kilic and R. Mahbubani, "Precision electroweak observables in the minimal moose little Higgs model," arXiv:hep-ph/0312053

[27] M. C. Chen and S. Dawson, arXiv:hep-ph/0311032.

[28] W. Kilian and J. Reuter, arXiv:hep-ph/0311095.

[29] C. x. Yue and W. Wang, "The branching ratio R(b) in the littlest Higgs model," Nucl. Phys. B 683, 48 (2004) arXiv:hep-ph/0401214.

[30] H. C. Cheng and I. Low, arXiv:hep-ph/0405243.

[31] S. R. Coleman, J. Wess and B. Zumino, "Structure Of Phenomenological Lagrangians. 1," Phys. Rev. 177 (1969) 2239.

[32] C. G. . Callan, S. R. Coleman, J. Wess and B. Zumino, "Structure Of Phenomenological Lagrangians. 2," Phys. Rev. 177 (1969) 2247.

[33] J. Thaler and I. Yavin, to appear.

[34] G. F. Giudice and A. Strumia, Nucl. Phys. B 663, 377 (2003) arXiv:hep-ph/0301232. 